\title{
Investigation of the growth patterns of non-functioning pituitary macroadenomas using volumetric assessments on serial MRI investigations
}

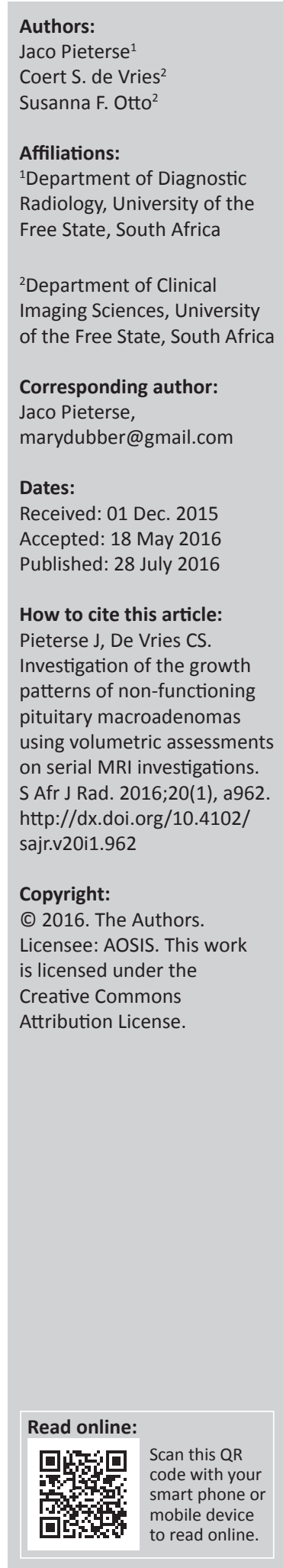

Background: Benign non-functioning pituitary macroadenomas (NFMA) often cause mass effect on the optic chiasm necessitating transsphenoidal surgery to prevent blindness. However, surgery is complicated and there is a high tumour recurrence rate. Currently, very little is known about the natural (and residual post-surgical) growth patterns of these NFMA. Conflicting data describe decreased growth to exponential growth over various time periods. Due to lack of information on growth dynamics of these NFMA, suitable follow-up imaging protocols have not been described to date.

Objective: To determine if NFMA grow or stay quiescent over a time period using serial MRI investigations and a stereological method to determine tumour volume. In addition, to evaluate if NFMA adhere to a certain growth pattern or grow at random.

Method: Thirteen patients with NFMA had serial MRI investigations over a 73-month period at the Universitas Academic Hospital. Six of the selected patients had undergone previous surgery, while seven patients had received no medical or surgical intervention. By using a stereological method, tumour volumes were calculated and plotted over time to demonstrate growth curves. The data were then fitted to tumour growth models already described in literature in order to obtain the best fit by calculating the $r^{2}$ value.

Results: Positive tumour growth was demonstrated in all cases. Tumour growth patterns of nine patients best fitted the exponential growth curve while the growth patterns of three patients best fitted the logistic growth curve. The remaining patient demonstrated a linear growth pattern.

Conclusion: A specific growth model best described tumour growth observed in non-surgical and surgical cases. If follow-up imaging confirms positive growth, future growth can be predicted by extrapolation. This information can then be used to determine the relevant follow-up-imaging interval in each individual patient.

\section{Introduction}

Pituitary adenomas are almost always benign (>99.9\%), arise from the anterior pituitary and account for approximately $15 \%$ of all intra-cranial tumours. ${ }^{1,2}$ The majority of these adenomas show no local invasion of bone, cavernous sinus or vascular encasement and malignant transformation and metastases are extremely rare ( $0.2 \%$ of all adenoma cases) $)^{2,3}$

Pituitary adenomas can be divided into microadenomas $(<1 \mathrm{~cm})$ or macroadenomas $(>1 \mathrm{~cm})$ according to their widest diameter. ${ }^{1,4}$ The prevalence of pituitary adenomas in the general population has been reported to be $10 \%-27 \%$, with macroadenomas considerably lower at $0.2 \%{ }^{5}$ Macroadenomas are predominantly non-functioning $(>80 \%),{ }^{5,6}$ that is, there is no clinical and biochemical evidence of pituitary hormonal overproduction and serum prolactin levels of $\leq 100 \mathrm{ng} / \mathrm{mL}$ are required for classification as such. ${ }^{1,5}$

Non-functioning pituitary macroadenomas (NFMA) are the most prevalent pituitary neoplasm and their growth is haphazard with lobulation. ${ }^{7}$ Growth normally occurs through the diaphragma sellae into the suprasellar cistern with a characteristic 'snowman' appearance. ${ }^{1}$

Clinically, patients present with visual field defects, headache or hypopituitarism due to NFMA mass effect on surrounding structures. ${ }^{2,4,5}$ It is well accepted that once visual disturbances are present, transsphenoidal surgery is required to prevent blindness. ${ }^{6,8}$ Although up to $80 \%$ of 
patients experience significant visual improvement postsurgery, ${ }^{6,8}$ it is a technically difficult procedure with a high tumour recurrence rate of $12 \%-46 \%{ }^{4,5}$ Multi-lobulated macroadenomas with vertical suprasellar expansion are less likely to be totally removed than round tumours. ${ }^{9}$ Apoplexy is another possible complication seen in approximately $5 \%$ of cases. ${ }^{10}$ It is defined as infarct or haemorrhage in the pituitary gland, most likely due to alterations in perfusion pressure. ${ }^{2,10}$

Incidentally detected pituitary adenomas represent $20 \%-$ $40 \%$ of all NFMA., ${ }^{3,7}$ Asymptomatic non-functioning macroadenomas incidentally found on imaging pose a treatment dilemma. In the absence of visual symptoms, the neurosurgeon may opt for an expectant approach although complications like apoplexy, blindness and hypopituitarism may occur. ${ }^{5}$ In addition, the time interval between imaging and performing surgery on these patients remains unclear. ${ }^{8}$

A meta-analysis by Ezzat et al. found that the prevalence of pituitary adenomas is $14.4 \%$ in autopsy studies and $22.5 \%$ in radiological studies. ${ }^{11}$

Due to uncertainty regarding growth of pituitary adenomas, various authors have attempted to study their growth over a selected time period. In a series of pituitary adenomas detected incidentally, Donovan and Corenblum, ${ }^{7}$ found positive growth in 4 of 16 large tumours ( $>1 \mathrm{~cm}$ in height) but no growth in 15 tumours smaller than $1 \mathrm{~cm}$ in height over 6.1 and 6.7 years, respectively. This suggests that a small percentage show considerable growth but the majority remain stable. Tumour enlargement thus seems more predominant in macroadenomas compared with microadenomas. ${ }^{11}$ Reincke et al. ${ }^{12}$ also noted an increase in tumour height in three of 14 incidentalomas monitored over 22 months. Average tumour height was $13 \mathrm{~mm}$ with a range of 5 to $25 \mathrm{~mm}$ in diameter.

Dekkers et al. ${ }^{8}$ investigated the natural growth course of NFMA. Growth was defined as a $0.01 \mathrm{~cm}$ increase in size in any direction and positive growth was shown in 14 of the 28 cases. They used an ellipsoid formula to approximate tumour volume but unfortunately this method does not allow for the measurement of irregular edges. In addition, a spontaneous decrease in tumour volume was reported in $29 \%$ of patients.

Growth of postoperative residual adenomas has also been studied. Turner et al. ${ }^{13}$ observed tumour regrowth in 21 of 65 patients over a 76-month follow-up period.

However, in all of the mentioned studies; however, heterogeneous criteria were used for defining tumour growth. Tumour height was seen as the most important predictor of optic chiasm compression resulting in calculations based on tumour height only. ${ }^{3}$ Other authors calculated initial and end tumour volumes with no description of the actual growth pattern. Thus, these studies failed to provide suitable quantitative information on the actual growth velocity of pituitary macroadenomas.
Tanaka et al. ${ }^{14}$ used the first volumetric assessment technique to calculate tumour volume by scanning the tumour outline on a computer. Regrowth was seen in 38 of 40 residual postsurgical tumours mostly in an exponential fashion.

Honneger et al. ${ }^{11}$ performed the first quantitative assessment of growth on incidentalomas in post-surgical cases. They used the stereological method to calculate tumour volume based on calculating tumour area on each contiguous slice. They found statistically significant exponential tumour growth in the majority of cases which enabled them to suggest a follow-up imaging protocol for patients.

Based on the results from studies done to date, the stereological method is the most accurate way to assess tumour volumes on imaging because it accommodates the haphazard growth often observed.

In this study, the NFMA growth pattern and rate was determined in a South African population using the stereological method used by Honneger et al. ${ }^{11}$ Measurements were, however, taken in two planes from thin MRI slices to increase measurement accuracy. The growth patterns observed were then fitted to mathematical growth models described in literature.

\section{Research method and design}

Approval was obtained from the University of the Free State Ethics Committee as well as the National Health Research Database in the Free State prior to study commencement.

Retrospective data were collected from patients who received follow-up MRIs between 29 October 2008 and 10 November 2014 at the Universitas Academic Hospital in Bloemfontein. Suitable cases were chosen from the information available from 46 patients on the database. Informed consent was therefore not required for this study. Subjects were assigned random numbers and are referred to as such.

Patient selection was based on benign macroadenoma appearance on imaging regardless of age, race or sex. Histological confirmation of pituitary macroadenoma was required in post-surgical cases. NFMA also had to be classified as non-functional in order to ensure that dopamine antagonists were not concomitantly used, which might have caused a decrease in tumour size. Potential patients were therefore required to have a baseline serum prolactin level of below $100 \mathrm{ng} / \mathrm{mL}$ and these results were obtained from the University National Health Laboratory Service (NHLS).

Serial MRI investigations of 13 patients ( 3 women and 10 men) from the age of 25 years to 69 years who had all the necessary evaluations done according to the above criteria, were studied over a 73-month period. Vertical tumour measurement had to be more than $1 \mathrm{~cm}$ and all patients had received between three and six serial MRI investigations. 
Six of these patients had undergone initial adenoma surgery, and only follow-up imaging was used for calculations. Post-surgery imaging was typically done within 3 months after the initial procedure. The remaining seven patients had received no medical or surgical intervention. The decision to withhold surgery was made by the managing clinicians and reasons included patients declining surgery, carotid artery encasement and poor anaesthetic risk. Patients with apoplexy were excluded as this could have influenced tumour volume.

The average tumour volume was calculated from the measurements in two planes (sagittal and coronal). The primary researcher did the measurements on two separate occasions under the direct supervision of a neuroradiologist. The average of these two values was then used to calculate the in-depth volumetric assessment according to the stereological model.

\section{Materials}

All MRI investigations were obtained with a 1.5 tesla MRI machine (General Electric, Signa Dual Gradient, Milwaukee, USA).

\section{Procedure}

Thin MRI slices in the coronal and sagittal planes were studied in all cases. All patients received intravenous gadolinium at a dose of $0.2 \mathrm{~mL} / \mathrm{kg}$.

A standard imaging protocol was used for all patients:

- Field of view $=20 \mathrm{~mm}^{2}$

- Matrix = Frequency $320 \times$ Phase 224

- Slice thickness between 2 and $5.0 \mathrm{~mm}$

- Spacing between 0.2 and $0.5 \mathrm{~mm}$

- Number of excitations $=5$.

A stereological method was used to obtain tumour volumes in both sagittal and coronal planes. Tumour edges were outlined by free-hand on each slice and then the respective two-dimensional area was calculated using an imaging processing software program (Isite) available on the Patient Archive and Communication System (PACS). Tumour volume was calculated on standard radiology reporting monitors.

Tumour area (measured in $\mathrm{cm}^{2}$ ) on each slice was then multiplied by the sum of the slice thickness and the intervening gap to calculate the volume of one slice. All values were then added to estimate the total tumour volume for each serial MRI investigation. All tumour volumes were measured in $\mathrm{cm}^{3}$ and all time periods were recorded in days.

The data were captured into Microsoft Excel before submission to the Department of Biostatistics for further statistical analysis.

\section{Analyses}

Average tumour volume of the sagittal and coronal planes was plotted using at least four consecutive data points and fitted to the following three established mathematical models:

- Logistic: Initially exponential growth followed by a linear growth phase and finally approaching a constant plateau.

- Exponential: During a period of time there is a constant percentage increase in tumour volume.

- Linear: Constant increase in tumour volume.

The 'goodness of fit' was obtained by calculating the square of the correlation coefficient $\left(r^{2}\right.$ value). For patients with insufficient data points, the $r^{2}$ value could not be calculated. Growth was nonetheless described based on the shape of the observed curve when plotted using the available points.

\section{Results}

Figure 1 demonstrates six serial T1 weighted post-contrasted MRI slices through the tumour in the coronal plane. As demonstrated in Figure $2 a$ and Figure $2 b$, these tumours often have lobulated outer margins.

All subjects demonstrated positive tumour growth over the observation period (Table 1). The initial measured median tumour volume was $11.96 \mathrm{~cm}^{3}$ compared to an end median volume of $19.00 \mathrm{~cm}^{3}$ after the 73-month period.

In nine cases, NFMA growth was best demonstrated by an exponential curve. Of these, three had sufficient data points to calculate the $r^{2}$ value. Higher $r^{2}$ values for the exponential model (mean $r^{2}=0.99$ ) were found compared with the logistic model (mean $r^{2}=0.95$ ). No statistical significance was found when comparing the exponential to the logistic growth patterns $(p=0.06)$. Observed growth that best fitted the exponential model had an $r^{2}$ value of 0.97 (Figure 3). Of the nine cases, three had received surgery and six had been treated conservatively. The initial mean volume was $16.24 \mathrm{~cm}^{3}( \pm 10.15)$ and end volume was $25.69 \mathrm{~cm}^{3}( \pm 16.03)$. The minimum initial tumour volume was $2.68 \mathrm{~cm}^{3}$ and maximum tumour end volume was $52.08 \mathrm{~cm}^{3}$.

The logistic model best described three cases, with only two patients having sufficient data points to calculate the $r^{2}$ value. Of these, a mean $r^{2}$ value of 0.94 was seen compared with a mean $r^{2}$ value of 0.85 in the exponential model. No statistical significance was found on comparing the logistic and exponential growth patterns $(p=0.37)$. Observed growth that best fitted the logistic growth model had an $r^{2}$ value of 0.94 (Figure 4). In two of the cases, the patients had received surgery and, in one case, the patient had received conservative treatment only. The initial mean volume was 


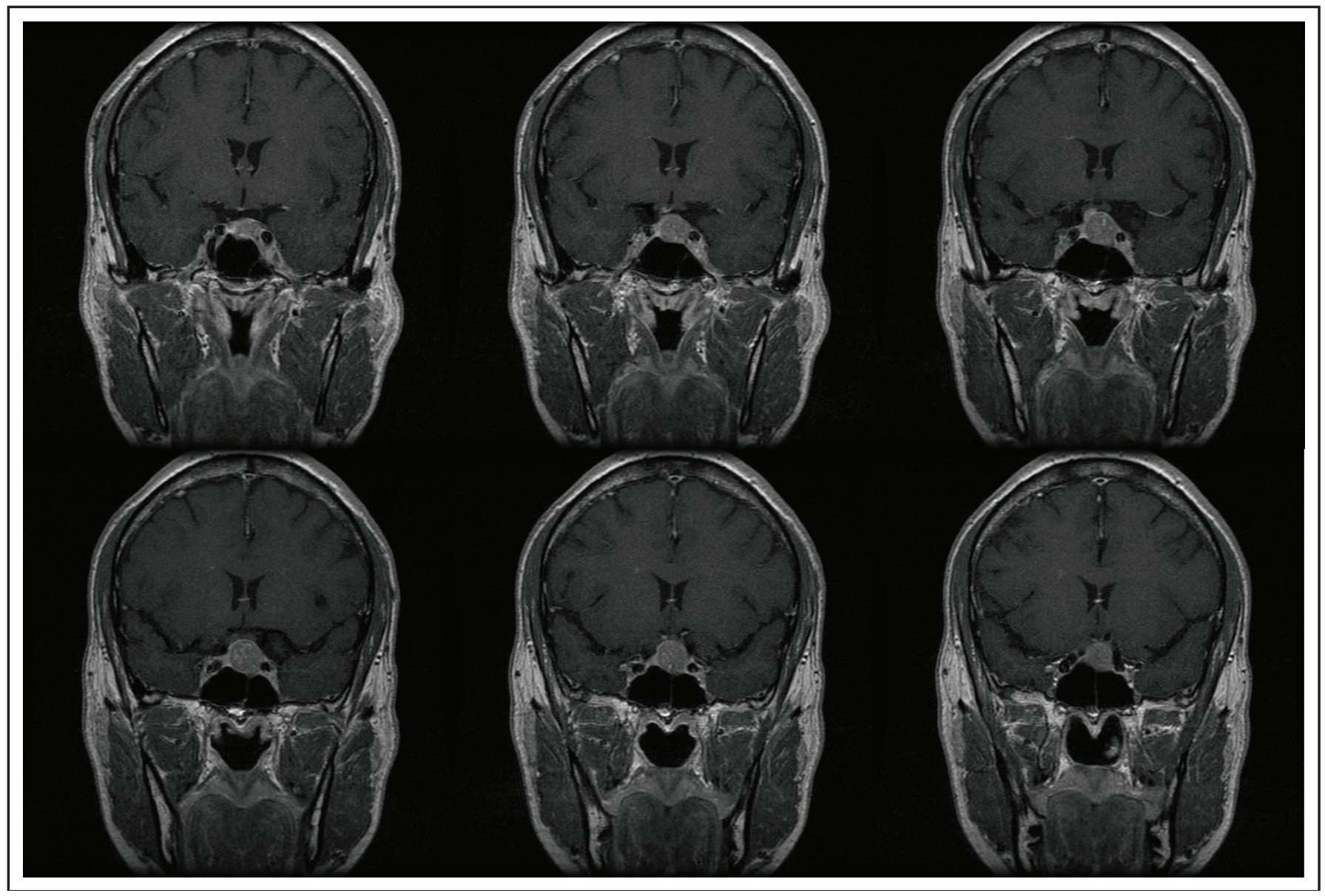

Note: Coronal T1-weighted (post-contrast) slices through the tumour. Slice space was $0.22 \mathrm{~cm}$ and the slice gap $0.02 \mathrm{~cm}$ Source: PACS image from 3 megapixel BARCO reporting monitor at the Universitas Academic Hospital FIGURE 1: Non-functioning pituitary macroadenoma in a 46-year-old male.

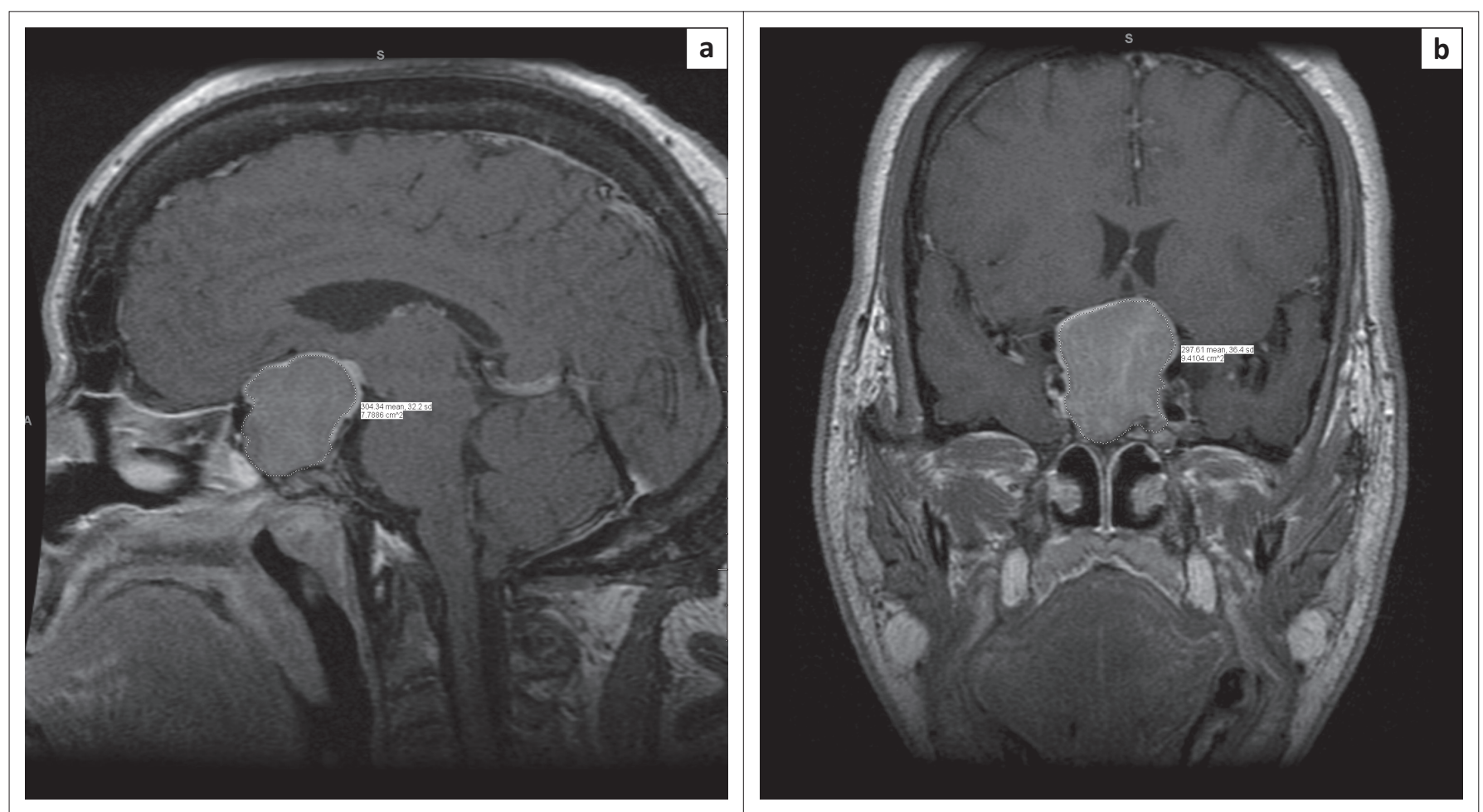

Note: Outline is lobulated with suprasellar extension and the characteristic 'snowman' appearance. The images demonstrate the area tracings.

Source: PACS image from 3 megapixel BARCO reporting monitor at the Universitas Academic Hospital

FIGURE 2: Large non-functioning pituitary macroadenoma in a 54-year-old male: Sagittal (a) and coronal (b) T1-weighted images (post-contrast) of the brain. 
TABLE 1: Clinical data, imaging studies as well as growth patterns observed during the observation period.

\begin{tabular}{|c|c|c|c|c|c|c|c|c|c|c|c|}
\hline \multirow{2}{*}{$\begin{array}{l}\text { Patient } \\
\text { number }\end{array}$} & \multirow[t]{2}{*}{ Age } & \multirow[t]{2}{*}{ Sex } & \multirow[t]{2}{*}{ Surgery } & \multirow[t]{2}{*}{ Serial MRIs } & \multirow{2}{*}{$\begin{array}{c}\text { Observation } \\
\text { period (days) }\end{array}$} & \multicolumn{4}{|c|}{ Volumetric assessment $\uparrow$} & \multirow{2}{*}{$\begin{array}{l}\text { Growth pattern } \\
\text { observed }\end{array}$} & \multirow{2}{*}{$\begin{array}{c}\text { Goodness of fit } \\
(\%)\end{array}$} \\
\hline & & & & & & $\begin{array}{l}\text { Initial volume } \\
\text { average }\left(\mathrm{cm}^{3}\right)\end{array}$ & $\begin{array}{l}\text { End volume } \\
\text { average }\left(\mathrm{cm}^{3}\right)\end{array}$ & $\begin{array}{c}\text { Volume } \\
\text { difference }\left(\mathrm{cm}^{3}\right)\end{array}$ & $\begin{array}{c}\text { Growth per } \\
\text { year }\left(\mathrm{cm}^{3} / \text { year }\right)\end{array}$ & & \\
\hline 1 & 46 & Male & No & 3 & 473 & 2.68 & 3.42 & 0.75 & 0.58 & Exponential & $\mathrm{n} / \mathrm{a}$ \\
\hline 2 & 69 & Male & Yes & 4 & 861 & 5.54 & 7.13 & 1.59 & 0.68 & Logistic & $93.73 * *$ \\
\hline 3 & 42 & Male & No & 3 & 490 & 15.17 & 18.9 & 3.73 & 2.78 & Exponential & $\mathrm{n} / \mathrm{a}$ \\
\hline 4 & 66 & Male & Yes & 5 & 1512 & 11.32 & 14.06 & 2.74 & 0.66 & Exponential & $99.28 *$ \\
\hline 5 & 54 & Male & Yes & 3 & 735 & 21.68 & 52.08 & 30.4 & 15.1 & Exponential & $\mathrm{n} / \mathrm{a}$ \\
\hline 6 & 61 & Male & No & 3 & 875 & 18.45 & 30.4 & 11.95 & 4.98 & Exponential & $\mathrm{n} / \mathrm{a}$ \\
\hline 7 & 52 & Female & Yes & 3 & 2016 & 2.35 & 7.01 & 4.66 & 0.84 & Linear & $\mathrm{n} / \mathrm{a}$ \\
\hline 8 & 60 & Female & No & 3 & 408 & 38.67 & 41.72 & 3.05 & 2.73 & Exponential & $\mathrm{n} / \mathrm{a}$ \\
\hline 9 & 25 & Female & No & 4 & 475 & 0.7 & 0.77 & 0.07 & 0.05 & Logistic & $93.74 * *$ \\
\hline 10 & 40 & Male & No & 3 & 616 & 9.82 & 12.8 & 2.98 & 1.76 & Exponential & $\mathrm{n} / \mathrm{a}$ \\
\hline 11 & 46 & Male & Yes & 3 & 1603 & 0.76 & 0.86 & 0.1 & 0.02 & Logistic & $\mathrm{n} / \mathrm{a}$ \\
\hline 12 & 46 & Male & No & 4 & 1457 & 17.89 & 18.49 & 0.59 & 0.15 & Exponential & $97.39 *$ \\
\hline
\end{tabular}

$\dagger$, Based on average volume in the sagittal and coronal planes; ${ }^{*}, p=0.06$ when compared to logistic model; **, $p=0.37$ when compared to exponential model; n/a, not applicable.

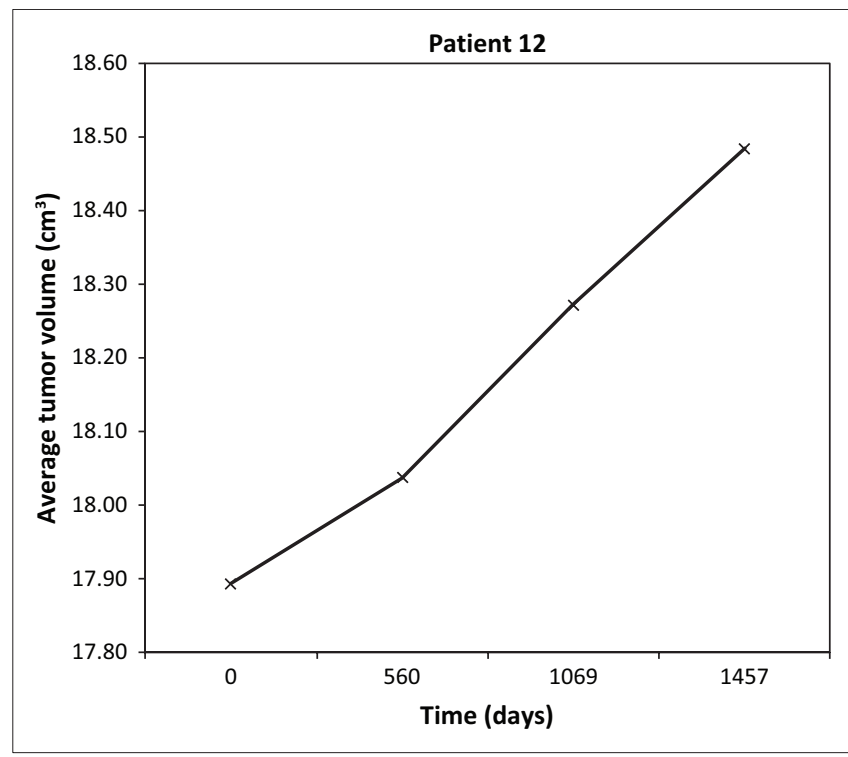

Note: The $r^{2}$ of $97.39 \%$ adheres to the best fit of the exponential growth pattern. FIGURE 3: Macroadenoma growth observed in a 46-year-old male.

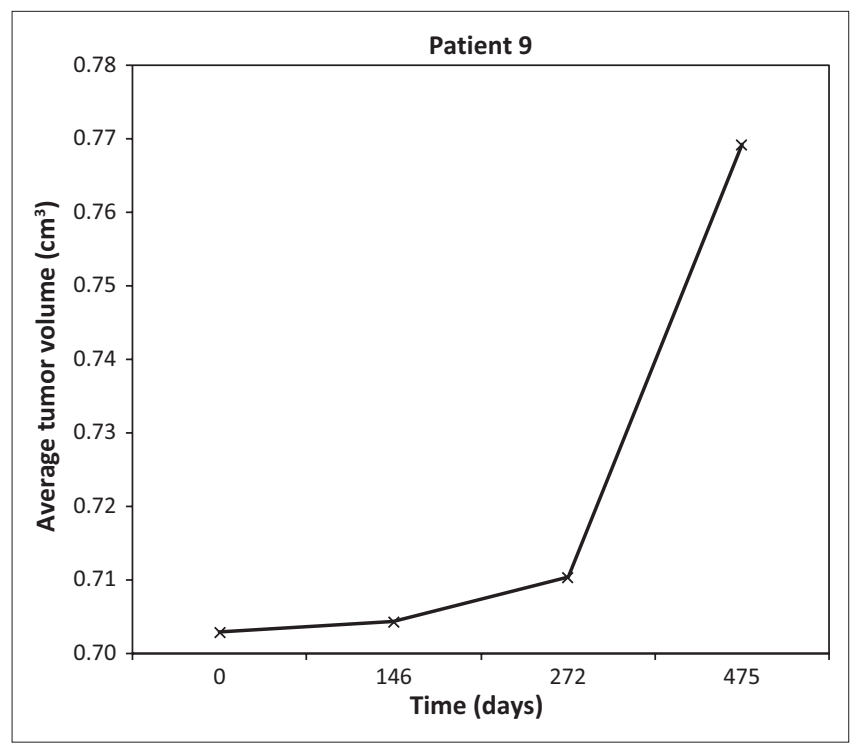

Note: The $r^{2}$ value of $93.74 \%$ adheres to the best fit of the logistic growth pattern. FIGURE 4: Macroadenoma growth observed in a 25 -year-old female.
$2.33 \mathrm{~cm}^{3}( \pm 2.77)$ and end volume was $2.92 \mathrm{~cm}^{3}( \pm 3.64)$. The minimum initial volume was $0.70 \mathrm{~cm}^{3}$ and maximum end volume was $7.13 \mathrm{~cm}^{3}$.

Only one case exhibited a linear trend; however, there were insufficient data points to calculate the $r^{2}$ value. This patient had received surgical intervention. Initial tumour volume was $2.35 \mathrm{~cm}^{3}$, and end volume was $7.01 \mathrm{~cm}^{3}$.

In adherence to exponential growth, the five largest tumours, with an initial calculated volumes of more than $15 \mathrm{~cm}^{3}$ demonstrated a higher average growth rate of $5.15 \mathrm{~cm}^{3} /$ year compared with $1.10 \mathrm{~cm}^{3} /$ year seen in tumours smaller than $15 \mathrm{~cm}^{3}$. Furthermore, tumours adhering to the exponential growth pattern had an average initial size of $16.24 \mathrm{~cm}^{3}$ compared with $2.33 \mathrm{~cm}^{3}$ seen in the logistic pattern.

\section{Discussion}

Mathematical modelling has been applied to describe growth of other tumours in literature. Experimental tumour paradigms of malignant and rapidly growing tumours typically adhere to a sigmoidal curve (Figure 5). This is described as the logistic mathematical model: an exponential phase followed by a linear phase and lastly, a plateau phase. Final deceleration is likely due to impaired blood supply with central necrosis and restricted growth in the peripheral regions. ${ }^{11}$

However, in contrast to malignant tumours, NFMA are benign, homogenous and solid, and it is hypothesised that they should show stable growth behaviour. If the entire volume of a solid tumour grows at a constant rate, exponential tumour growth would be expected resulting in a constant tumour volume doubling time. ${ }^{11}$

All the patients in this study showed an increase in tumour volume over time. It seems that when volumetric assessment is applied on high quality imaging, the incidence of growth is much higher compared with previous studies. On follow-up imaging, the tumours 


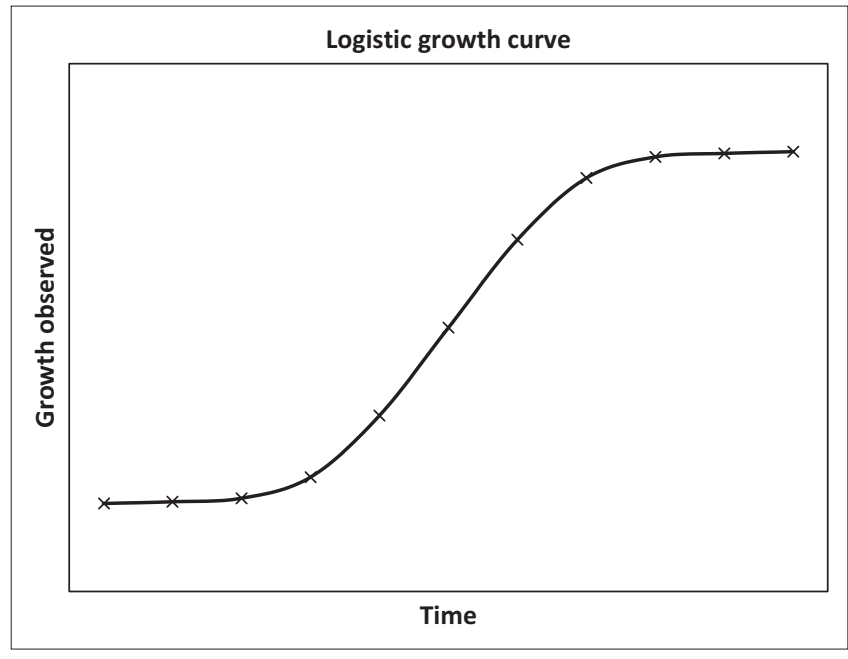

FIGURE 5: Logistic growth curve representing growth observed in malignant tumours.

often appeared unchanged in size only to find a significant volume increase after the necessary calculations. This contrasts the spontaneous decrease in tumour volume reported in $29 \%$ of patients in the study by Dekker et al. ${ }^{11}$ A decrease in volume is, however, highly improbable due to the solid nature of these tumours.

No clear trend in the tumour growth patterns could be deducted for conservatively treated adenomas versus postsurgical cases or in different age groups.

Observations of this study were similar to those of Honegger et al., ${ }^{11}$ with the majority of cases (69\%) adhering to the exponential growth model. In the five cases with enough data points, high $r^{2}$ values for the exponential and logistic models were obtained, but the $p$-value was not statistically significant to classify growth into a specific model. In cases with insufficient data points, the graph trend was used to classify growth to a specific growth model.

Larger initial mean NFMA $\left(16.24 \mathrm{~cm}^{3}\right)$ tend to show exponential growth when compared with smaller tumours $\left(2.33 \mathrm{~cm}^{3}\right)$, which exhibited logistic growth. On an individual case level, however, initial tumour size cannot be used to accurately determine the growth velocity or pattern (e.g. patient 1 who demonstrated exponential NFMA growth had an initial tumour volume of $2.68 \mathrm{~cm}^{3}$, and patient 2 who showed logistic NFMA growth had an initial volume of $\left.5.54 \mathrm{~cm}^{3}\right)$.

The hypothesis that adenomas with an exponential pattern would eventually follow a logistic growth pattern could not be determined due to the fixed observation period.

One patient demonstrated adherence to the linear model; however, it can be postulated that the NFMA might have been in the linear growth phase of the logistic model.
These results demonstrate that if there is a significant increase in growth at first follow-up, exponential or logistic growth can be expected in future. If no increase in growth is found, it is unlikely that the tumour will grow rapidly in the near future. Once the individual patient trends are established, appropriate and effective treatment can be prescribed for these patients, which includes a follow-up MRI-protocol tailored for each individual.

\section{Limitations of the study}

The lack of long-term follow-up due to the fixed observation period was seen as a study limitation. As a result, the possibility that these benign tumours would eventually demonstrate logistic growth could not be determined.

\section{Conclusion}

Based on these results, it can be recommended that radiologists calculate tumour volume using this stereological method on serial MRI imaging to determine the growth rate. In conjunction with imaging, regular follow-up regarding ophthalmology evaluation and hormonal assessment is advised.

This insight into the growth dynamics of NFMA may facilitate the development of a tailored follow-up imaging protocol for patients with either incidentalomas or post-surgical NFMA. This may reduce healthcare expenses by allowing an individualised rational treatment plan.

\section{Acknowledgements}

The first author would like to thank Dr Otto for reviewing the measurement process, Prof. De Vries for his guidance as study leader, the Department of Neurosurgery for providing the subject information, the National Health Laboratory Services for assisting me with the relevant blood results and the Department of Biostatistics at the UOFS.

\section{Competing interests}

The authors declare that they have no financial or personal relationships which may have inappropriately influenced them in writing this article.

\section{Authors' contributions}

C.S.d.V. was the project leader. J.P. performed the measurements and performed all the calculations and S.F.O. made conceptual contributions and supervised the measurement process.

\section{References}

1. Dekkers OM. Nonfunctioning pituitary macroadenomas: Treatment and long term follow-up. PhD Thesis. Universiteit Leiden; 2006.

2. Mamelak AN, Carmichael JD, Park P, Bannykh S, Fan X, Bonert HV. Atypical pituitary adenoma with malignant features. Pituitary 2011;14(1):92-97. http:// dx.doi.org/10.1007/s11102-008-0151-1 
3. Berkmann S, Schlaffer S, Buchfelder M. Tumor shrinkage after transsphenoidal surgery for nonfunctioning pituitary adenoma. J Neurosurg 2013;119(6):1447-1452. surgery for nonfunctioning pituitary adenoma.
$\mathrm{htt}$ d .doi.org/10.3171/2013.8.JNS13790

4. Arita K, Tominaga A, Sugiyama K, et al. Natural course of incidentally found nonfunctioning pituitary adenoma, with special reference to pituitary apoplexy during follow-up examination. J Neurosurg. 2006;104(6):884-891. http://dx.doi. org/10.3171/jns.2006.104.6.884

5. Dekkers OM, Pereira AM, Romijn JA. Treatment and follow-up of clinically nonfunctioning pituitary macroadenomas. J Clin Endocrinol Metab. 2008; 93(10):3717-3726. http://dx.doi.org/10.1210/jc.2008-0643

6. Turner HE, Stratton IM, Byrne JV, Adams CB, Wass JA. Audit of selected patient with nonfunctioning pituitary adenomas treated without irradiation - A followup study. Clin Endocrinol (Oxf) 1999;51(3):281-284. http://dx.doi.org/10.1046/ j.1365-2265.1999.00865.x

7. Honegger J, Ernemann U, Psaras T, Will B. Objective criteria for successful transsphenoidal removal of suprasellar nonfunctioning pituitary adenomas. A prospective study. Acta Neurochir (Wien). 2007;149(1):21-29. http://dx.doi. org/10.1007/s00701-006-1044-6

8. Dekkers OM, Hammer S, de Keizer RJW, et al. The natural course of nonfunctioning pituitary macroadenomas. Eur J Endocrinol. 2007;156(2):217-224. $\mathrm{http}: / / \mathrm{dx}$.doi.org/10.1530/eje.1.02334
9. Donovan LE, Corenblum B. The natural history of the pituitary incidentaloma. Arch Intern Med. 1995;155(2):181-183. http://dx.doi.org/10.1001/archinte. 1995.00430020067008

10. Lee J-S, Park Y-S, Kwon J-T, Nam T-K, Lee T-J, Kim J-K. Radiological apoplexy and its correlation with acute clinical presentation, angiogenesis and tumor microvascular density in pituitary adenomas. J Korean Neurosurg Soc. 2011:50(4):281-287. density in pituitary adenomas. J Korean Ne
$\mathrm{http}: / / \mathrm{dx}$.doi.org/10.3340/jkns.2011.50.4.281

11. Honegger J, Zimmermann S, Psaras T, et al. Growth modelling of non-functioning pituitary adenomas in patients referred for surgery. Eur J Endocrinol. 2008;158(3):287-294. http://dx.doi.org/10.1530/EJE-07-0502

12. Glass RM, Drummond W, Cole HM, et al. The 'incidentaloma' of the pituitary gland. Is neurosurgery required? J Am Med Assoc. 1990;263(20):2772-2776. http://dx.doi.org/10.1001/jama.1990.03440200076026

13. Anagnostis P, Adamidou F, Polyzos SA, Efstathiadou Z, Panagiotou A, Kita M. Nonfunctioning pituitary adenomas: A single center experience. Exp Clin Endocrinol Diabetes. 2011;119(5):314-319. http://dx.doi.org/10.1055/s-0030-1267991

14. Tanaka Y, Hongo K, Tada T, Sakai K, Kakizawa Y, Kobayashi S. Growth pattern and rate in residual nonfunctioning pituitary adenomas: Correlations among tumor volume doubling time, patient age, and MIB-1 index. J Neurosurg. 2003;98(2):359-365. http://dx.doi.org/10.3171/jns.2003.98.2.0359 